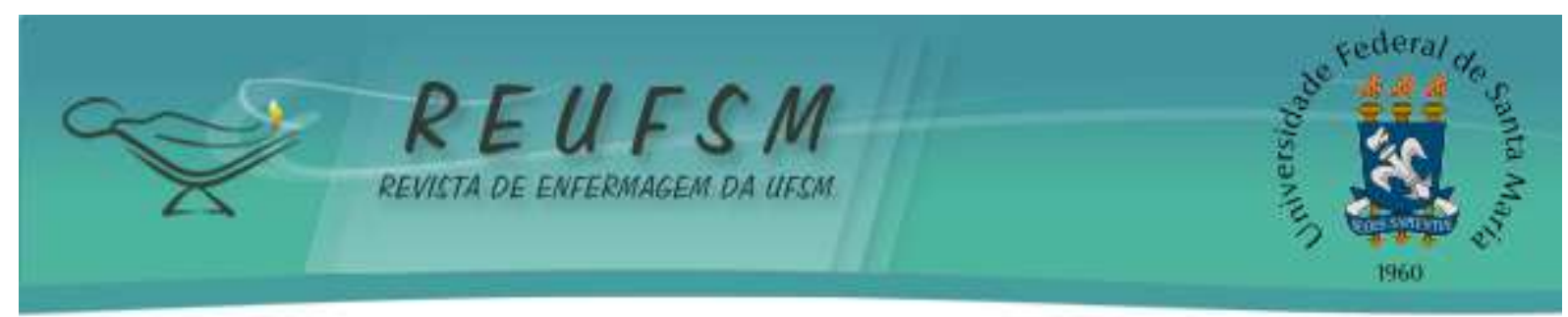

ARTIGO ORIGINAL

\title{
PERFIL SOCIODEMOGRÁFICO E CLÍNICO DE PACIENTES COM ÚLCERAS VENOSAS ACOMPANHADOS EM AMBULATÓRIO: ESTUDO TRANSVERSAL DESCRITIVO
}

\section{SOCIODEMOGRAPHIC AND CLINICAL PROFILE OF PATIENTS WITH VENOUS ULCERS IN OUTPATIENT FOLLOW-UP: DESCRIPTIVE CROSS-SECTIONAL STUDY}

\section{PERFIL SOCIODEMOGRÁFICO Y CLÍNICO DE PACIENTES CON ÚLCERAS VENOSAS ACOMPAÑADOS EN CLÍNICA: ESTUDIO DESCRIPTIVO TRANSVERSAL}

\author{
Thaís Dresch Eberhardt ${ }^{1}$ \\ Suzinara Beatriz Soares de Lima ${ }^{2}$ \\ Luis Felipe Dias Lopes ${ }^{3}$ \\ Jocelaine Cardoso Gracióli ${ }^{4}$ \\ Graziele Gorete Portella da Fonseca ${ }^{5}$ \\ Lorena Foureaux Ribeiro ${ }^{6}$
}

Doi: $10.5902 / 2179769223054$

RESUMO: Objetivo: identificar o perfil sociodemográfico e clínico dos pacientes com úlceras venosas, acompanhados no ambulatório de um hospital universitário do sul do Brasil. Método: estudo transversal descritivo, realizado em um ambulatório de angiologia de um hospital universitário. Os dados foram coletados de 21 pacientes com úlceras venosas, de março a julho de 2015, por meio de um formulário; e analisados por meio de estatística descritiva simples. Resultados: os participantes do estudo, em sua maioria, estavam na faixa etária entre 64 e 72 anos, eram do sexo masculino, da raça branca e aposentados, apresentavam uma úlcera venosa, com período inferior a um ano de existência e sem recidivas. Conclusão: os pacientes com úlcera venosa são, em sua maioria, homens idosos, com uma úlcera venosa recente e sem recidivas.

DESCRITORES: Enfermagem; Úlcera varicosa; Perfil de saúde; Assistência ambulatorial.

ABSTRACT: Aim: to identify the sociodemographic and clinical profile of patients, who were outpatients from a university hospital in southern Brazil and that had venous ulcers. Method: this is a descriptive and transversal survey conducted in a angiology clinic of a university hospital. Data were collected through form, from 21 patients with venous ulcers, from March to July 2015 and analyzed by means of simple descriptive statistics. Results: the participants were mostly aged between 64 and 72, male, caucasian and retired. Each one of them had a venous ulcer with less than one year of existence and no relapses. Conclusion: patients with venous ulcers are mostly elderly men with a recent venous ulcers without recurrence.

DESCRIPTORS: Nursing; Varicose ulcer; Health profile; Ambulatory care.

\footnotetext{
${ }^{1}$ Enfermeira, Mestre em Enfermagem, Doutoranda do Programa de Pós-Graduação em Enfermagem, Universidade Federal de Santa Maria, Santa Maria, Rio Grande do Sul, Brasil. E-mail: thaiseberhardt@gmail.com ${ }^{2}$ Enfermeira, Doutora em Enfermagem, Professora Adjunta, Universidade Federal de Santa Maria, Santa Maria, Rio Grande do Sul, Brasil. E-mail: suzibslima@yahoo.com.br

${ }^{3}$ Bacharel em Matemática, Doutor em Engenharia da Produção, Professor Associado, Universidade Federal de Santa Maria, Santa Maria, Rio Grande do Sul, Brasil. E-mail: Iflopes67@yahoo.com.br

${ }^{4}$ Enfermeira, Mestranda do Programa de Pós-Graduação em Enfermagem, Universidade Federal de Santa Maria, Santa Maria, Rio Grande do Sul, Brasil. E-mail: jocegraci@ yahoo.com.br

${ }^{5}$ Enfermeira, Mestranda do Programa de Pós-Graduação em Enfermagem, Universidade Federal de Santa Maria, Santa Maria, Rio Grande do Sul, Brasil. E-mail: grazieleportelladafonseca@gmail.com

${ }^{6}$ Enfermeira, Santa Maria, Rio Grande do Sul, Brasil. E-mail: lorenafoureaux@yahoo.com.br
} 


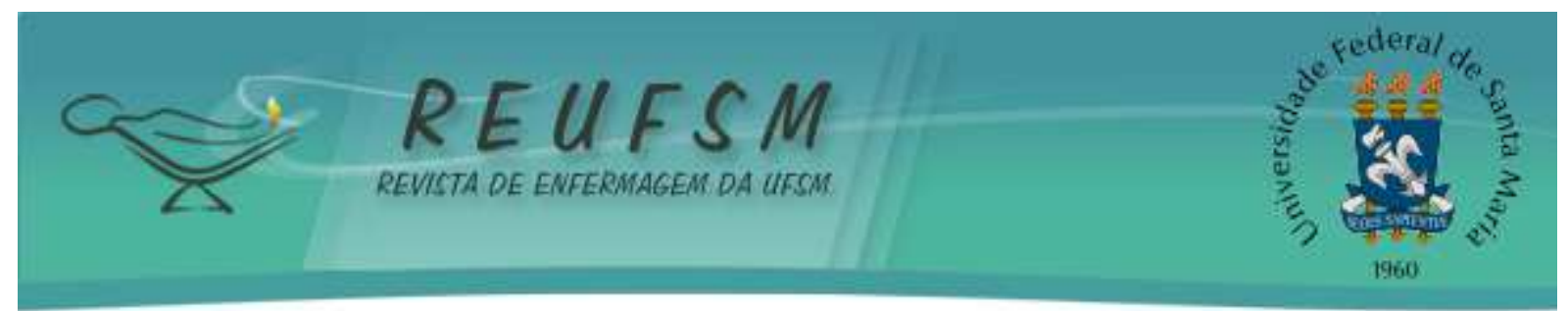

RESUMEN: Objetivo: identificar el perfil sociodemográfico y clínico de los pacientes con úlceras venosas ambulatorios en un hospital universitario, en el sur de Brasil. Método: estudio descriptivo transversal realizado en una clínica de angiología de un hospital universitario. Se recolectó datos de 21 pacientes con úlceras venosas, entre marzo y julio de 2015, por medio de encuestas; y se analizóa partir deestadísticas descriptivas sencillas. Resultados: los participantes del estudio, tenían en la mayoría entre 64 y 72 años, eran del sexo masculino, de raza caucásica y jubilados, tenían unaúlcera venosa, a menos de un año y no habían presentado recaídas. Conclusión: los pacientes con úlceras venosas son la mayoría hombres, ancianos, con un reciente úlceras venosas sin recidiva.

DESCRIPTORES: Enfermería; Úlcera varicosa; Perfil de salud; Atención ambulatoria.

\section{INTRODUÇÃO}

A ferida crônica é uma lesão na pele com baixa ou nenhuma probabilidade de cicatrizar, que se mantém durante a causa que a produz. Esta possui uma etiologia subjacente que transforma a cicatrização em um processo longo, como, por exemplo, a úlcera venosa. ${ }^{1}$

Devido à natureza recorrente, incapacitante e que necessita de terapêutica prolongada, estas lesões causam elevado custo ao sistema de saúde, constituindo-se em um problema de saúde pública. ${ }^{1}$

Entre as úlceras de perna, a úlcera venosa (UV) é a que apresenta maior prevalência, correspondendo em torno de 80 a $90 \%$ dos casos. ${ }^{2}$ Além disso, podem causar impacto significativo na vida dos pacientes, devido à dor crônica, desconforto, depressão, redução da autoestima, isolamento social, inabilidade para o trabalho, necessidade de hospitalizações e frequente acompanhamento ambulatorial. ${ }^{3}$

Desta forma, a enfermagem possui atuação próxima às pessoas acometidas por estas lesões, pois realiza assistência na avaliação, planejamento e realização de curativos; solicita avaliação e encaminhamento a outros profissionais para assistirem o paciente; bem como atua como educador em saúde.

Nesse contexto, este estudo permitirá maior conhecimento sobre os aspectos sociodemográficos, assistenciais e clínicos das pessoas, com a finalidade de auxiliar os profissionais de enfermagem no planejamento do gerenciamento do cuidado destes pacientes em cenário ambulatorial.

Em uma revisão da literatura sobre a produção brasileira de teses e dissertações desenvolvidas pela enfermagem na temática da cicatrização, encontrou-se como lacuna o perfil de pacientes com feridas atendidos na atenção secundária e terciária. ${ }^{4}$ Com base nessa revisão e no contexto apresentado, justifica-se a realização deste estudo.

A partir do exposto, elaborou-se a seguinte questão de pesquisa: "Quais as características sociodemográficas e clínicas dos pacientes com UV acompanhados no ambulatório de um hospital universitário do sul do Brasil? Assim, tem-se como objetivo identificar o perfil sociodemográfico e clínico dos pacientes com úlceras venosas acompanhados no ambulatório de um hospital universitário do sul do Brasil.

\section{MÉTODO}

Trata-se de um estudo transversal descritivo, realizado em um ambulatório de angiologia de um hospital universitário do sul do Brasil. 


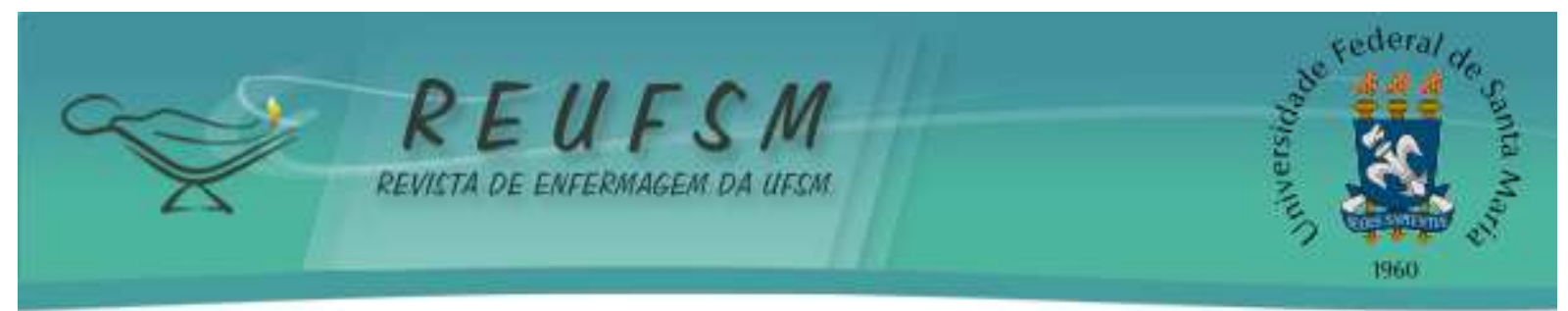

Para seleção dos participantes do estudo se utilizou como critério de inclusão: todos os pacientes com diagnóstico médico de UV, atendidos no ambulatório de angiologia de um hospital universitário do sul do Brasil no período de coleta de dados (março a julho de 2015) com idade igual ou superior a 18 anos. Foram excluídos pacientes com capacidade cognitiva ou comunicação verbal diminuída sem acompanhante, devido à dificuldade em responder ao formulário de coleta de dados; pacientes que possuíam úlcera cobrindo mais que uma face do membro inferior. ${ }^{i}$

Foram identificados 48 pacientes, destes, nove recusaram-se a participar do estudo, cinco foram excluídos, pois possuíam úlcera cobrindo mais que uma face do membro inferior, 11 não foram captados durante o acompanhamento, um desistiu, um apresentava capacidade de comunicação verbal prejudicada e estava sem acompanhante. Portanto, foram incluídos no estudo 21 pacientes com 36 UV.

Os dados demográficos e clínicos foram coletados por meio de formulário estruturado, preferencialmente do prontuário do paciente. No entanto, quando estes dados não constavam no prontuário, as perguntas foram respondidas pelo próprio paciente e/ou acompanhante.

As variáveis coletadas foram classificadas em:

a) sociodemográficas: idade, sexo, raça, escolaridade, estado civil, ocupação atual e renda mensal (na época da coleta de dados, o salário mínimo era de R\$ 788,00);

b) clínicas: índice de massa corporal e presença de morbidades;

c) quanto aos dados do membro afetado com UV: edema, veias varicosas, eritema, descamação, prurido, quantidade de úlceras, tempo de existência das lesões, presença de úlceras prévias e dor;

d) dados da UV: localização, profundidade, quantidade de exsudato, odor do exsudato, coloração do exsudato, tecido predominante presente no leito da ferida e presença de alteração nas bordas.

Os dados foram digitados no Microsoft Office Excel $\AA$, por meio de digitação dupla e independente, a fim de corrigir possíveis falhas de digitação. Após, foram transportados e analisados no Programa Estatístico R versão 3.2.2.

Os dados foram analisados por meio de estatística descritiva simples: as variáveis qualitativas com frequência absoluta e frequência relativa; as variáveis quantitativas com média e desvio padrão (DP) ou mediana e intervalo interquartil (IQ) conforme a normalidade dos dados.

Os aspectos éticos da pesquisa foram amparados pela Resolução do Conselho Nacional de Saúde $n^{\circ} 466$ de 12 de dezembro de 2012. O projeto foi submetido à apreciação de Comitê de Ética de Pesquisa, sendo aprovado sob o parecer $n^{\circ} 932.838$ e Certificado de Apresentação para Apreciação Ética $n^{\circ}$ 40250814.6.0000.5346. A pesquisa procedeu mediante assinatura pelos participantes do Termo de Consentimento Livre e Esclarecido.

\section{RESULTADOS}

Participaram do estudo 21 pessoas com diagnóstico de úlcera venosa, com idade média de $60,9(11,6)$ anos. A maioria $(66,7 \%)$ era do sexo masculino, raça branca $(66,6 \%)$, possuía ensino fundamental incompleto $(66,6 \%)$, era casada $(52,4 \%)$, aposentada $(71,4 \%)$ e possuía renda mensal per capita mediana de 1,0 salários mínimos (Tabela 1). 


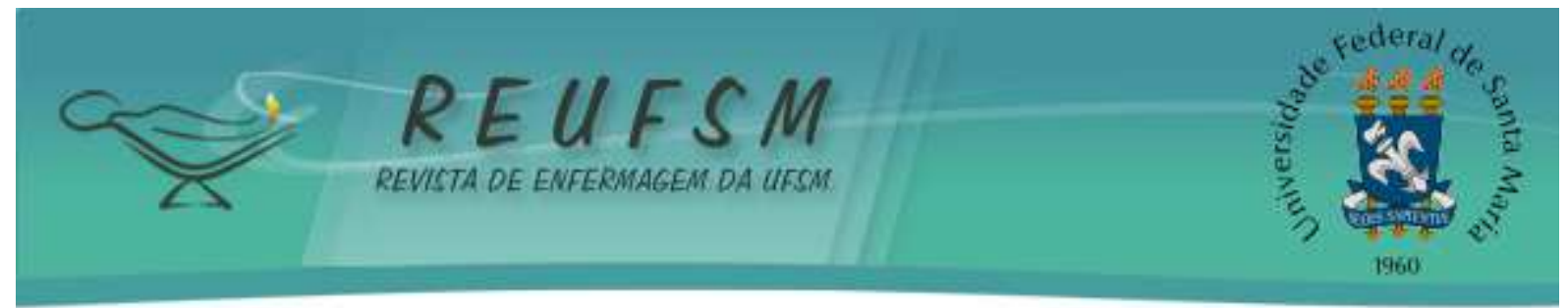

Tabela 1 - Dados sociodemográficos dos pacientes com úlcera venosa acompanhados ambulatorialmente. Santa Maria, RS, Brasil, 2015. $n=21$

\begin{tabular}{|c|c|c|}
\hline Variável & $\mathrm{n}$ & $\%$ \\
\hline \multicolumn{3}{|l|}{ Faixa etária } \\
\hline $37-45$ & 2 & 9,5 \\
\hline $46-54$ & 4 & 19,0 \\
\hline $55-63$ & 5 & 23,8 \\
\hline $64-72$ & 9 & 42,9 \\
\hline $73-82$ & 1 & 4,8 \\
\hline \multicolumn{3}{|l|}{ Sexo } \\
\hline Feminino & 7 & 33,3 \\
\hline Masculino & 14 & 66,7 \\
\hline \multicolumn{3}{|l|}{ Raça } \\
\hline Branca & 14 & 66,6 \\
\hline Preto-negra & 1 & 4,8 \\
\hline Parda & 5 & 23,8 \\
\hline Amarela & 0 & 0,0 \\
\hline Indígena & 1 & 4,8 \\
\hline \multicolumn{3}{|l|}{ Escolaridade } \\
\hline Ensino fundamental incompleto & 14 & 66,6 \\
\hline Ensino fundamental completo & 3 & 14,3 \\
\hline Ensino médio incompleto & 3 & 14,3 \\
\hline Ensino médio completo & 1 & 4,8 \\
\hline \multicolumn{3}{|l|}{ Estado civil } \\
\hline Solteiro (a) & 3 & 14,3 \\
\hline União estável & 0 & 0,0 \\
\hline Casado (a) & 11 & 52,4 \\
\hline Divorciado (a) & 3 & 14,3 \\
\hline Viúvo (a) & 4 & 19,0 \\
\hline \multicolumn{3}{|l|}{ Ocupação atual } \\
\hline Aposentado (a) & 15 & 71,4 \\
\hline Outros & 6 & 28,6 \\
\hline \multicolumn{3}{|l|}{ Renda mensal (por pessoa) } \\
\hline < 1 salário mínimo & 3 & 14,3 \\
\hline 1 salário mínimo & 13 & 61,9 \\
\hline > 1 salário mínimo & 5 & 23,8 \\
\hline
\end{tabular}

$\mathrm{Na}$ Tabela 2, podem ser observados os dados clínicos dos pacientes. Estes possuíam índice de massa corporal (IMC) médio de $29,7(6,2) \mathrm{Kg} / \mathrm{m}^{2}$, quanto à presença de morbidades, a doença mais relatada foi a hipertensão arterial sistêmica $(66,7 \%)$, seguida da insuficiência venosa crônica (IVC) $(28,6 \%)$ e 18 (85,7\%) faziam uso contínuo de medicamentos.

Tabela 2 - Dados clínicos dos pacientes com úlcera venosa acompanhados ambulatorialmente. Santa Maria, RS, Brasil, 2015. $\mathrm{n}=21$

\begin{tabular}{ccc}
\hline Variável & $\mathbf{n}$ & $\%$ \\
\hline Índice de massa corporal $\left(\mathrm{Kg} / \mathrm{m}^{2}\right)$ & & \\
Abaixo do normal $(<18,5)$ & 0 & 0,0 \\
Adequado $(18,5-24,9)$ & 4 & 19,0 \\
Sobrepeso $(25,0-29,9)$ & 7 & 33,3 \\
Obesidade grau I $(30,0-34,9)$ & 6 & 28,6 \\
Obesidade grau II $(35,0-39,9)$ & 3 & 14,3 \\
Obesidade grau III $(>39,9)$ & 1 & 4,8 \\
Presença de morbidades & & \\
\hline
\end{tabular}




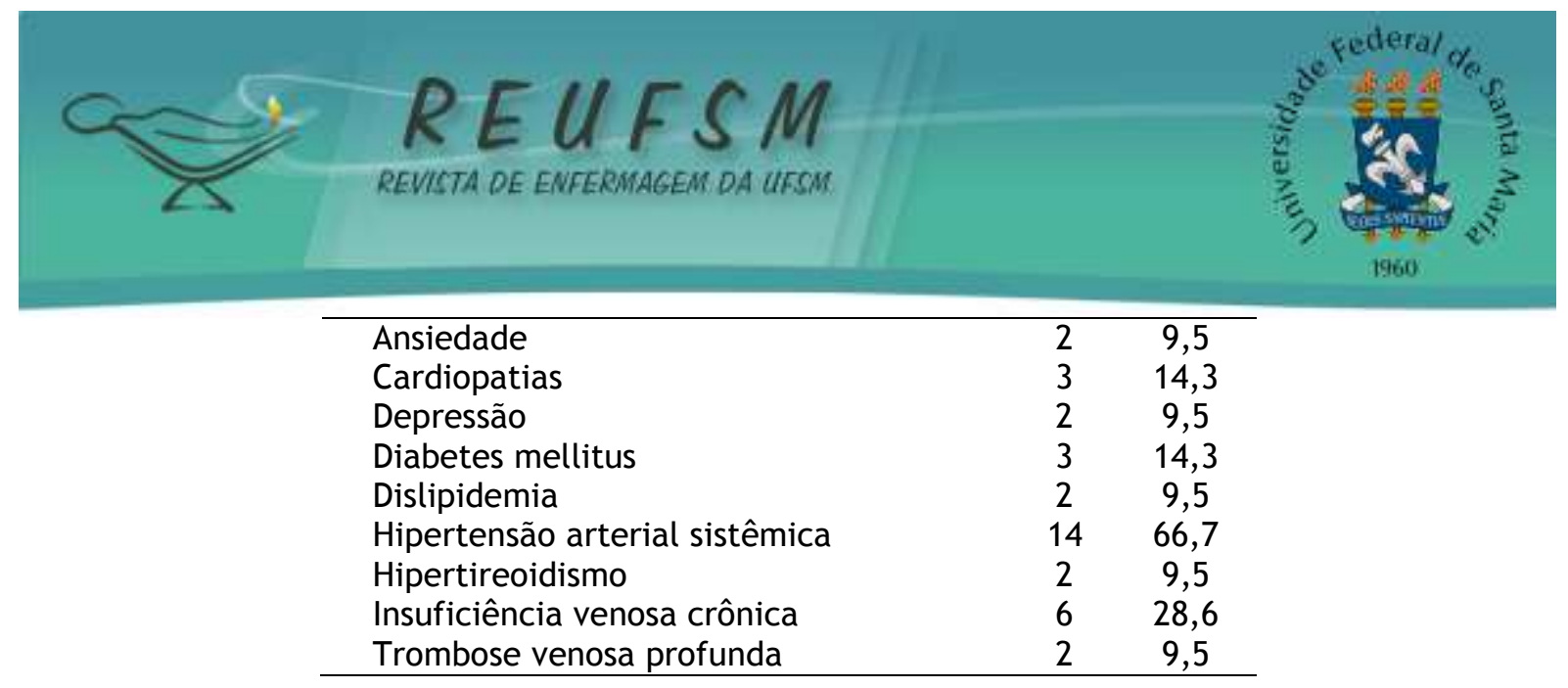

Os dados referentes ao membro afetado com a UV podem ser visualizados na Tabela 3. A maioria apresentava alteração (edema, veias varicosas, eritema, descamação e prurido). 0 número de UV variou entre uma e cinco por paciente, com mediana de 1,8 $(1,0-3,0)$. As lesões existiam a um tempo mediano de 3,0 (2,0-14,0) anos e $11(52,4 \%)$ não apresentavam úlceras prévias. A intensidade da dor apresentada foi variável, com média de $1,9(0,0-6,3)$ pontos.

Tabela 3 - Dados do membro afetado com úlcera venosa dos pacientes acompanhados ambulatorialmente. Santa Maria, RS, Brasil, 2015. $\mathrm{n}=21$

\begin{tabular}{|c|c|c|}
\hline Variável & $\mathbf{n}$ & $\%$ \\
\hline \multicolumn{3}{|l|}{ Presença de edema } \\
\hline Sim & 19 & 90,5 \\
\hline Não & 2 & 9,5 \\
\hline \multicolumn{3}{|l|}{ Presença de veias varicosas } \\
\hline Sim & 14 & 66,7 \\
\hline Não & 7 & 33,3 \\
\hline \multicolumn{3}{|l|}{ Presença de eritema } \\
\hline Sim & 21 & 100,0 \\
\hline Não & 0 & 0,0 \\
\hline \multicolumn{3}{|l|}{ Presença de descamação } \\
\hline Sim & 13 & 61,9 \\
\hline Não & 8 & 38,1 \\
\hline \multicolumn{3}{|l|}{ Presença de prurido } \\
\hline Sim & 11 & 52,4 \\
\hline Não & 10 & 47,6 \\
\hline \multicolumn{3}{|l|}{ Quantidade de úlceras } \\
\hline 1 & 13 & 61,9 \\
\hline 2 & 3 & 14,3 \\
\hline 3 & 4 & 19,0 \\
\hline 4 & 0 & 0,0 \\
\hline 5 & 1 & 4,8 \\
\hline \multicolumn{3}{|c|}{ Tempo de existência das lesões (anos) } \\
\hline$<1$ ano & 22 & 9,5 \\
\hline $1-10$ anos & 12 & 57,2 \\
\hline$>11$ anos & 7 & 33,3 \\
\hline \multicolumn{3}{|l|}{ Presença de úlceras prévias } \\
\hline Sim & 10 & 47,6 \\
\hline Não & 11 & 52,4 \\
\hline \multicolumn{3}{|l|}{ Dor $^{1}$} \\
\hline Ausência de dor (0) & 6 & 28,6 \\
\hline Leve $(1-4)$ & 8 & 38,1 \\
\hline Moderada (5-7) & 2 & 9,5 \\
\hline Intensa $(8-10)$ & 5 & 23,8 \\
\hline
\end{tabular}

1: Dor segundo a Escala Visual Analógica. 


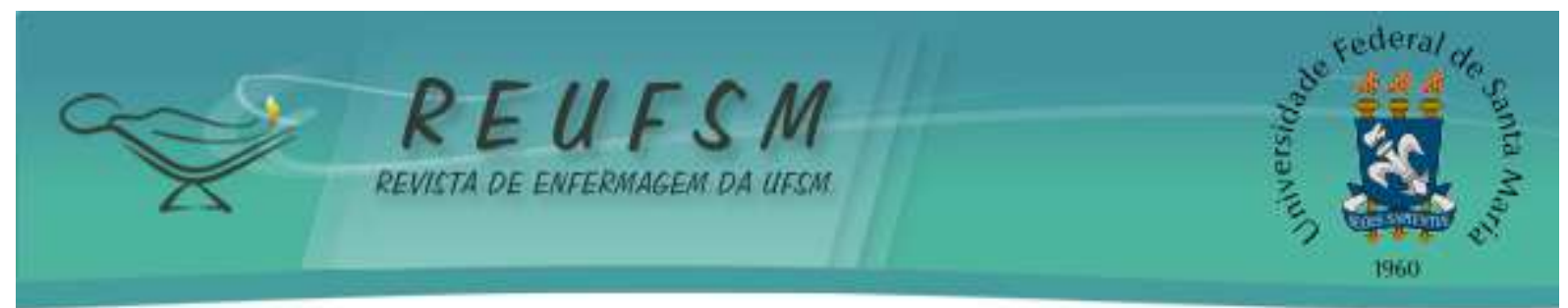

$\mathrm{Na}$ tabela 4, são apresentados os dados referentes às úlceras venosas ( $\mathrm{n}=36$ ). Quanto à localização, 26 (72,3\%) feridas estavam na região II (metade proximal da perna até abaixo do tornozelo), $25(69,5 \%)$ apresentavam perda parcial de tecidos. Em relação ao exsudato, $27(75,0 \%)$ apresentavam quantidade mínima, 32 (88,9\%) sem odor e $25(69,5 \%)$ possuíam aspecto seroso. A maioria das feridas apresentava como tecido predominante em seu leito o esfacelo $(86,1)$ e também alguma alteração nas bordas $(77,8 \%)$.

Tabela 4 - Dados das úlceras venosas dos pacientes acompanhados ambulatorialmente. Santa Maria, RS, Brasil, 2015. $\mathrm{n}=36$

\begin{tabular}{|c|c|c|}
\hline Variável & $\mathbf{n}$ & $\%$ \\
\hline \multicolumn{3}{|l|}{ Localização1 } \\
\hline Região I & 7 & 19,4 \\
\hline Região II & 26 & 72,3 \\
\hline Região III & 3 & 8,3 \\
\hline \multicolumn{3}{|l|}{ Profundidade } \\
\hline Perda parcial de tecidos & 25 & 69,5 \\
\hline Perda total de tecidos & 11 & 30,5 \\
\hline \multicolumn{3}{|l|}{ Quantidade de exsudato } \\
\hline Ferida ressecada & 1 & 2,8 \\
\hline Mínima & 27 & 75,0 \\
\hline Moderada & 6 & 16,7 \\
\hline Máxima & 2 & 5,5 \\
\hline \multicolumn{3}{|l|}{ Odor do exsudato } \\
\hline Ausente & 32 & 88,9 \\
\hline Fétido & 4 & 11,1 \\
\hline \multicolumn{3}{|l|}{ Coloração do exsudato } \\
\hline Seroso & 25 & 69,5 \\
\hline Sanguinolento & 4 & 11,1 \\
\hline Serossanguinolento & 4 & 11,1 \\
\hline Piossanguinolento & 2 & 5,5 \\
\hline Esverdeado & 1 & 2,8 \\
\hline \multicolumn{3}{|c|}{ Tecido presente predominante no leito da ferida } \\
\hline Tecido de granulação & 4 & 11,1 \\
\hline Necrose de coagulação & 1 & 2,8 \\
\hline Esfacelo & 31 & 86,1 \\
\hline \multicolumn{3}{|l|}{ Presença de alteração nas bordas } \\
\hline Sim & 28 & 77,8 \\
\hline Não & 8 & 22,2 \\
\hline
\end{tabular}

1: Região I - pé; Região II - metade proximal da perna até abaixo do tornozelo; Região III - metade distal da perna até a borda inferior da patela. Fonte: o autor.

\section{DISCUSSÃO}

No presente estudo, a idade média dos participantes foi de 60,9 anos, indicando um alerta para a equipe de saúde, pois, com o envelhecimento, aumentam as morbidades crônicas e degenerativas de saúde e, com elas, os desafios econômicos e estruturais da saúde pública. . $^{-6}$

Observou-se predomínio de participantes do sexo masculino, assemelhando-se a outro estudo realizado no estado de Goiás. ${ }^{7} \mathrm{Em}$ contrapartida, há outros cenários relatados, ${ }^{8-10}$ nos quais é evidenciado predomínio do sexo feminino. As mulheres procuram mais os serviços de saúde para prevenção dos agravos, o que poderia explicar essa situação, uma vez que, normalmente, os homens procuram assistência diante da presença de complicações, como a UV. 


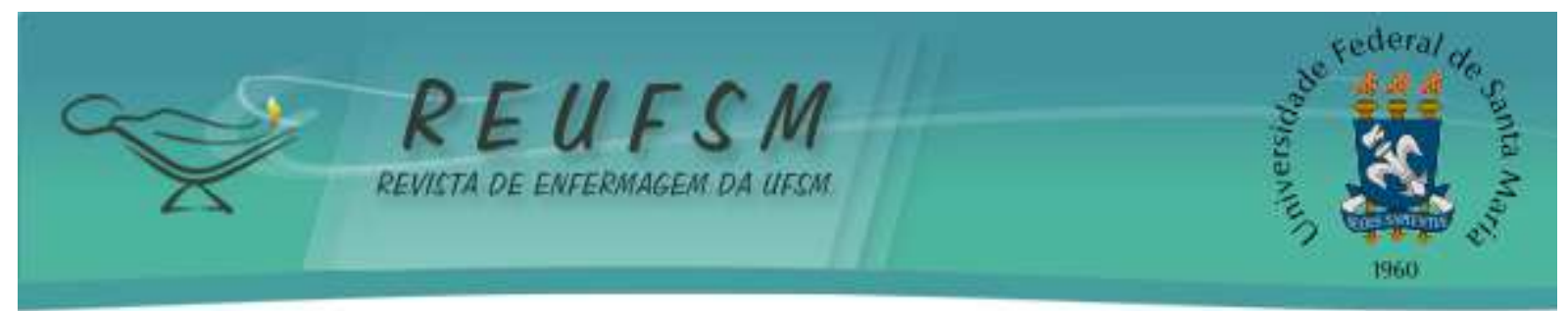

Observou-se baixo nível de escolaridade, que pode estar relacionado à capacidade de compreensão das orientações sobre o processo do autocuidado, ação necessária para o sucesso do tratamento; também ao acesso aos serviços de saúde e aos recursos materiais. ${ }^{10-11}$

Quanto ao estado civil, os resultados se assemelham aos de outro estudo, em que $60 \%$ das pessoas com UV se declararam casados ou vivendo uma união estável, o que se entende como positivo, uma vez que o(a) companheiro(a) acompanha o paciente com úlcera em suas necessidades diárias e o incentiva para o tratamento.${ }^{6,8,10} \mathrm{~A}$ raça predominante foi a branca, o que pode estar associada à região do país de colonização europeia.

Em concordância com outros estudos, a maioria das pessoas com UV possui renda mensal de um salário mínimo. ${ }^{7-8}$ Este fato pode influenciar na continuidade do tratamento que, por vezes, é oneroso, principalmente quando o material para a terapêutica não é distribuído nos serviços de saúde. ${ }^{12-13}$

Corroborando outras pesquisas, a maioria dos pacientes $(81,0 \%)$ estava acima do peso. ${ }^{8-9}$ Diante deste fato, a educação em saúde com o objetivo de controle do sobrepeso e obesidade é importante, incentivando-se a prática de atividades físicas. ${ }^{8}$

A IVC foi relatada por $28,6 \%$ dos pacientes deste estudo. Sabe-se que a IVC propicia a hipertensão venosa, aumenta a permeabilidade capilar, causando extravasamento de moléculas e acúmulo de líquido favorecendo o aparecimento da UV. ${ }^{14}$ Portanto, aponta-se a necessidade de atuação gerencial objetivando conhecer o motivo da ausência deste diagnóstico ou do registro desse diagnóstico no prontuário da maioria dos pacientes.

Ainda, ressalta-se que os registros adequados em prontuários são importantes para o acompanhamento do paciente. ${ }^{15}$ No que se refere aos registros, pesquisas ${ }^{16-18}$ mostram que é preciso melhorar a precisão e a abrangência nos registros.

Os sinais clínicos (edema, veias varicosas, eritema, descamação e prurido), encontrados nos membros afetados pelas UV, são um conjunto de indicadores clínicos que identificam alteração da circulação venosa. ${ }^{7,11}$ A maioria $(61,9 \%)$ dos pacientes apresentava uma UV, com tempo mediano de 3,0 anos de existência e sem recidivas, corroborando os achados de outro estudo. ${ }^{11}$

A ausência de dor foi relatada por $28,6 \%$ dos participantes. Outras pesquisas discutem a dor como um sintoma presente entre estes pacientes, podendo causar importante impacto na vida da pessoa e de seus familiares. ${ }^{5,19-20}$ Esses indivíduos podem, ainda, se isolar socialmente, perder a autonomia, perder a autoestima e desenvolver depressão, refletindo negativamente no tratamento. ${ }^{7,11,21}$

No que se refere à localização, identificou-se que a maioria das úlceras estava localizada na Região II (metade proximal da perna até abaixo do tornozelo) com profundidade superficial (perda parcial de tecidos). Estes dados se confirmam em literatura da área. ${ }^{3}$

A maior parte das lesões apresentava exsudato em quantidade mínima, sem odor e de aspecto seroso. É importante ressaltar que o exsudato seroso se apresenta como um processo natural em lesões crônicas, significando ausência de infecção no leito da ferida. ${ }^{11}$

0 esfacelo presente no leito das úlceras foi predominante e as bordas apresentavam algum tipo de alteração. As características do tecido do leito da lesão são importantes indicadores do estágio de cicatrização.

\section{CONCLUSÃO}

A presente pesquisa possibilitou a identificação do perfil sociodemográfico e clínico dos pacientes com úlcera venosa acompanhados ambulatorialmente. Percebeu-se que estes 


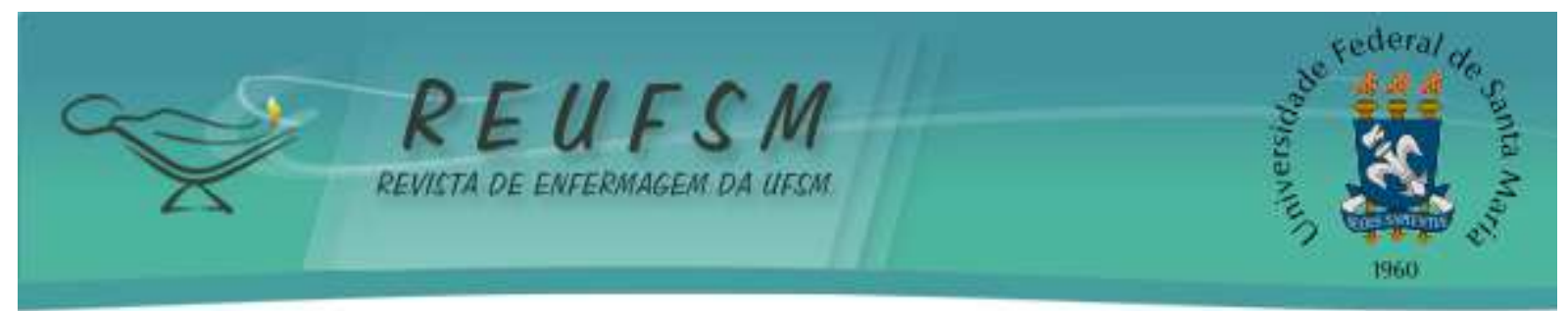

pacientes são, em sua maioria, homens idosos, da cor branca, casados, com baixo grau de escolaridade, aposentados, com renda mensal per capita de um salário mínimo.

Quanto às condições clínicas associadas, apresentavam morbidades como a hipertensão arterial sistêmica, insuficiência venosa crônica, além de sobrepeso. Ainda, alterações no membro afetado, uma úlcera venosa com menos de um ano de existência sem recidivas e dor leve. A úlcera estava localizada na região entre a metade proximal da perna até abaixo do tornozelo, com profundidade superficial, presença de quantidade mínima de exsudato seroso sem odor, leito da ferida com esfacelo e alteração nas bordas.

As limitações deste estudo se referem ao tipo de amostragem (não-probabilística) e à dificuldade em acessar aos pacientes que não retornaram às consultas. Sugere-se a realização de novos estudos que contemplem essa temática em diferentes cenários e populações. A caracterização dos pacientes com úlceras venosas, além de possuir relevância para o gerenciamento do cuidado de enfermagem, poderá contribuir com a construção de protocolos de pesquisa com metodologias mais específicas e detalhadas.

\section{REFERÊNCIAS}

1. Garcia Fernandez FP, López Casanova P, Segovia Gómez T, Soldevilla Agreda JJ, Verdú Soriano J; Grupo Nacional para el Estudio y Asesoramiento em Úlceras por Presión y Heridas Crónicas. Unidades multidisciplinares de heridas crónicas: clínicas de heridas. Logrono: GNEAUPP; 2012. (Documentos de Posicionamiento GNEAUPP; 10).

2. Guimarães Barbosa JA, Nogueira Campos LM. Diretrizes para o tratamento da úlcera venosa. Enferm Glob. 2010;(20):1-13.

3. Borges EL, organizadora. Feridas: úlceras dos membros inferiores. Rio de Janeiro: Guanabara Koogan; 2011.

4. Eberhardt TD, Dias CFC, Fonseca GGP, Kessler M, Soares RSA, Lima SBS. Cicatrização de feridas: análise das tendências em teses e dissertações. Rev Enferm UFSM. 2015;5(2):387-95.

5. Souza DMTS, Borges FR, Juliano Y, Veiga DF, Ferreira LM. Quality of life and self-esteem of patients with chronic ulcers. Acta Paul Enferm. 2013;26(3):283-8.

6. Torres GV, Costa IKF, Medeiros RKS, Oliveira AKA, Souza AJG, Mendes FRP. Caracterização das pessoas com úlcera venosa no Brasil e Portugal: estudo comparativo. Enferm Glob. 2013;12(32):62-74.

7. Malaquias SG, Bachion MM, Santana SMSC, Dallarmi CCB, Souza LJR, Ferreira OS. People with vascular ulcers in outpatient nursing care: a study of sociodemographic and clinical variables. Rev Esc Enferm USP. 2012;46(2):302-10.

8. Budó MLD, Durgante VL, Rizzatti SJS, Silva DC, Leal TC. Sociodemographic and health characterization of people with venous ulcers in outpatient clinic care. Rev Enferm UFPE online. 2013;7(3):731-7.

9. Borges EL, Amorim IPG, Carvalho DV. Características dos pacientes com úlcera venosa atendidos nas unidades de atenção primária de Nova Lima, Minas Gerais. Rev Estima. 2014;12(1):31-41.

10. Medeiros ABA, Andriola IC, Fernandes MICD, Silva FBBL, Sá JD, Lira ALBC. Socioeconomic profile of people with venous ulcer: relevant issues for nursing. Rev Enferm UFPE online. 2013;7(8):5220-4. 


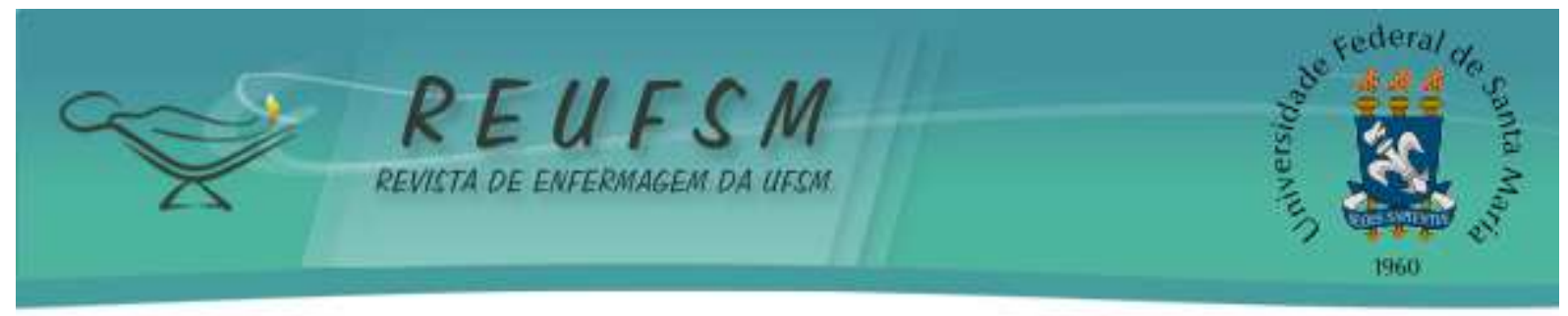

11. Oliveira BGRB, Castro JBA, Granjeiro JM. Panorama epidemiológico e clínico de pacientes com feridas crônicas tratados em ambulatório. Rev Enferm UERJ. 2013;21(Esp 1):612-7.

12.Dias TYAF, Costa IKF, Liberato SMD, Souza AJG, Mendes FRP, Torres GV. Quality of life for venous ulcer patients: a comparative study in Brazil/Portugal. Online Braz J Nurs. 2013;12(3):491-500.

13. Cardozo GM, Bermudes JPS, Araújo LO, Moreira ACMG, Ulbrich EM, Balduino AFA, et al. Contribuições da enfermagem para avaliação da qualidade de vida de pessoas com úlceras de perna. Rev Estima. ANO;10(2):19-27.

14. Nogueira GA, Oliveira BGRB, Santana RF, Cavalcanti ACD. Diagnósticos de enfermagem em pacientes com úlcera venosa crônica: estudo observacional. Rev Eletrônica Enferm. 2015;17(2):333-9.

15. Conforth A. Holistic wound assessment in primary care. $\mathrm{Br} \mathrm{J}$ Community Nurs. 2013:S28-34.

16. Thoroddsen A, Siqurjonsdóttir G, Ehnfors M, Ehrenberg A. Accuracy, completeness and comprehensiveness of information on pressure ulcers recorded in the patient record. Scand J Caring Sci. 2013;27(1):84-91.

17. Thompson N, Gordey L, Bowles H, Parlows N, Houghton P. Reliability and validity of the revised photographic wound assessment tool on digital images taken of various types of chronic wounds. Adv Skin Wound Care. 2013;26(8):360-73.

18. Li D, Korniewicz DM. Determination of the effectiveness of electronic health records to document pressure ulcers. Medsurg Nurs. 2013;22(1):17-25.

19. Santos LSF, Camacho ACLF, Oliveira BGRB, Nogueira GA, Joaquim FL. Influence of venous ulcer in patients' quality of life: an integrative review. Rev Enferm UFPE on line. 2015;9(3):7710-22.

20. Budó MLD, Durgante VL, Rizzatti SJS, Silva DC, Gewehr M, Farão EMD. Úlcera venosa, índice tornozelo braço e dor nas pessoas com úlcera venosa em assistência no ambulatório de angiologia. Rev Enferm Cent-Oeste Min. 2015;5(3):1794-804.

21. Costa LM, Higino WJF, Leal FJ, Couto RC. Clinical and socio-demographic profile of patients with venous disease treated in health centers of Maceió (AL), Brazil. J Vasc Bras. 2012;11(2):108-13.

Data de recebimento: 10/07/2016

Data de aceite: $21 / 12 / 2016$

Contato com autor responsável: Thaís Dresch Eberhardt

Endereço postal: Rua Elpídio Menezes n 21 apto. 106, Bairro Camobi, Santa Maria, Rio

Grande do Sul, Brasil, CEP 97105-110.

E-mail: thaiseberhardt@gmail.com

\footnotetext{
'Este estudo é proveniente de dissertação de mestrado "Mensuração de área de úlceras venosas por meio dos softwares AutoCAD® e Image Tool: reprodutibilidade de métodos"”, na qual foram realizadas fotografias das úlceras venosas. Por este motivo, as úlceras não poderiam cobrir mais que uma face do membro inferior, pois impossibilitaria a realização da fotografia e consequente mensuração da área.
} 